\title{
Predictions of the Particle Size Distribution Changes in Emulsions and Suspensions by Digital Computation
}

\author{
AKIRA SUZUKI, N. F. H. HO, AND W. I. HIGUCHI \\ College of Pharmacy, University of Michigan, Ann Arbor, Michigan \\ Received May 27, 1968; revised September 3, 1968
}

\begin{abstract}
The fundamental Smoluchowski flocculation rate equations including the effects of polydispersity and particle-particle potential energy barrier have been solved for any initial particle size distribution. The procedure for the numerical solution of the set of nonlinear differential equations involved the combined use of the Runge-Kutta and the Hamming methods by the IBM 360 computer. It was assumed that the only rate process is the passage of the particles over the primary electrical barrier to flocculation of suspensions (or coalescence of emulsion droplets). The input data were the surface potential, dielectric constant, temperature, Debye-Huckel kappa, viscosity, Hamaker constant, and the initial particle size distribution. The output included the time changes in the particle size distribution, the polydispersity and interaction barrier effeets on the rate and the time dependency of the mean polydispersity and interaction barrier of the dispersed system. The changes in the particle size distribution have also been studied by means of a similarity transformation which leads to a self-preserving spectrum. When the electrical barrier was small, any given initial distribution became more polydispersed with time and the total number of particles decreased more rapidly than second order with respect to time owing to the preferential flocculation of Müller. When the electrical barrier was appreciable, the distribution narrowed with time. These results were consistent with the particle size effects on the interaction potential. The predictions can now be directly used to compare experimental Coulter counter data with theory. The general method of numerical analysis can be easily adapted to cases involving other forces of repulsion between suspension and emulsion particles such as steric (or entropic) repulsion.
\end{abstract}

The general problem of theoretically predicting the particle size distribution changes with time for suspensions and emulsions due to perikinetic flocculation and/or immediate coalescence of emulsion droplets upon contact has been undertaken usually for the case in which no repulsive barriers are present (1-3) and to some extent for the case when there is an electrical repulsive barrier (4). The changes in the particle size distribution have also been studied by means of a similarity transformation which leads to a self-preserving spectrum $(2,3,5,6)$.

Recently, the problem of explicitly accounting for the particle size effects upon the particle-particle interactions was dis-

1 Tanabe Seiyaku, Osaka, Japan. cussed and some computations were presented (7). When electrical barriers exist between particles in a heterodispersed system, computations over a wide range of conditions have shown that small particles may preferentially flocculate (or coalesce) with themselves or with larger particles at rates that are 10 to 50 orders of magnitude faster than those of particles 10 times larger.

In the present study the fundamental flocculation equations including both the effects of polydispersity and interparticle potential energy barrier have been solved for any initial particle size distribution. It is noteworthy that many of the computations represent the first of their kind. It is now 
possible to directly relate time changes in particle size distribution of emulsions and suspensions to quantitative theories.

\section{THEORY}

The basic kinetic theory of rapid flocculation of colloidal particles was worked out by Smoluchowski (1). A refinement of the theory was given by Fuchs, who included the total interaction energy between two diffusing particles (1). The general flocculation expression, which is a set of nonlinear ordinary differential equations that describe the rate of change in the particle concentration of size $n_{k}$ in a discrete size distribution, is

$$
\begin{gathered}
\frac{d n_{k}}{d t}=\frac{1}{2} \sum_{i=1, j=k-i}^{i=k-1} \frac{4 \pi D_{i j} R_{i j} n_{i} n_{j}}{W_{i j}} \\
-n_{k} \sum_{i=1}^{\infty} \frac{4 \pi D_{i k} R_{i k} n_{i}}{W_{i k}} \\
(k=1,2,3 \cdots \infty),
\end{gathered}
$$

where $D_{i j}$ is the sum of the diffusivities of the $i$ th and $j$ th class particles, $R_{i j}$ is the collisional radius, $W_{i j}$ is the Fuchs stability function, $n_{i}, n_{j}$, and $n_{k}$ are the concentrations of the particles of class $i, j$, and $k$, respectively, and are time-dependent functions, and $t$ is the time. It is assumed that all particles are spheres and conjoin into spheres of an equivalent volume.

If $v_{i}$ is defined as

$$
\begin{aligned}
& v_{i}=i v_{1}, \\
& a_{i}=i^{1 / 3} a_{1},
\end{aligned}
$$

it follows that

$$
\begin{gathered}
D_{i j}=D_{i}+D_{j}=\frac{k T}{6 \pi \mu a_{1}\left(i^{1 / 3}+j^{1 / 3}\right)} ; \\
R_{i j}=a_{i}+a_{j}=a_{1}\left(i^{1 / 3}+j^{1 / 3}\right)^{.}
\end{gathered}
$$

Here $v$ and $a$ are the respective volume and radius of the particle, $\mu$ is the viscosity of the medium, $k$ is the Boltzmann constant, and $T$ is the absolute temperature, and the subscript 1 denotes the singlet particle. Equation [2] states that the volume of the $i$ th class particle is $i$ times that of the singlet particle.

The Smoluchowski flocculation half life
(1) is

$$
\tau=\left(\frac{4 k T N_{0}}{3 \mu}\right)^{-1}
$$

where $N_{0}$ is the total initial concentration of the particles. By defining the dimensionless variables $\sigma_{i j}, X$, and $Y_{k}$, we obtain

$$
\begin{aligned}
\sigma_{i j} & =1 / 4\left(i^{1 / 3}+j^{1 / 3}\right)\left(i^{-1 / 3}+j^{-1 / 3}\right) \\
X & =t / \tau \\
Y_{k} & =n_{k} / N_{0}
\end{aligned}
$$

and by substitution of Eqs. [4] to [9] into Eq. [1], the general expression becomes

$$
\begin{aligned}
\frac{d Y_{k}}{d X}=\sum_{i=1, j=k-i}^{i=k-1} \frac{\sigma_{i j} Y_{i} Y_{j}}{W_{i j}} & \\
& -2 \sum_{i=1}^{\infty} \frac{\sigma_{i k} Y_{i} Y_{k}}{W_{i k}} .
\end{aligned}
$$

In order to relate the fundamental theory, i.e., the rate of change of individual class particles as described by Eq. [1] or [10], to the usual treatment of experimental flocculation data, i.e., the rate of change of the total number of particles irrespective of size, it is useful to extend $\mathrm{Eq}$. [10] for all particle sizes; consequently,

$\frac{d \sum_{k=1}^{\infty} Y_{k}}{d X}=\frac{d Y_{\infty}}{d X}=-\sum_{i=1}^{\infty} \sum_{j=1}^{\infty} \frac{\sigma_{i j}}{W_{i j}} Y_{i} Y_{j}$.

Here $Y_{i}, Y_{j}$, and $Y_{\infty}$ are all dimensionless time-dependent functions. The mean value of $\sigma_{i j} / W_{i j}$ at time $X$ is

$$
\begin{aligned}
\left\langle\sigma_{i j} / W_{i j}\right\rangle_{X}=\frac{\sum_{i=1}^{\infty} \frac{\sum_{j=1}^{\infty} \frac{\sigma_{i j}}{W_{i j}} Y_{i} Y_{j}}{Y_{\infty}{ }^{2}}}{\left\langle\sigma_{i j} / W_{i j}\right\rangle_{X}>0,}
\end{aligned}
$$

and the total rate becomes

$$
\frac{d Y_{\infty}}{d X}=-\left\langle\sigma_{i j} / W_{i j}\right\rangle_{X} Y_{\infty}^{2}
$$

Thus, the time-dependent function $\left\langle\sigma_{i j} / W_{i j}\right\rangle_{x}$ accounts for the effect of polydispersity and the interaction barrier between particles on the flocculation rate. However, if one considers the mean values 
of $\sigma_{i j}$ and $1 / W_{i j}$ at time $X$, that is,

$$
\begin{gathered}
\left\langle\sigma_{i j}\right\rangle_{X}=\frac{\sum_{i=1}^{\infty} \frac{\sum_{j=1}^{\infty} \sigma_{i j} Y_{i} Y_{j}}{Y_{\infty}^{2}}}{\left\langle\sigma_{i j}\right\rangle_{X} \geqq 1 ;} \\
\left\langle 1 / W_{i j}\right\rangle_{X}=\frac{\sum_{i=1}^{\infty} \sum_{j=1}^{\infty} \frac{\sigma_{i j}}{\bar{W}_{i j}} Y_{i} Y_{j}}{\sum_{i=1}^{\infty} \sum_{j=1}^{\infty} \sigma_{i j} Y_{i} Y_{j}} \\
0<\left\langle 1 / W_{i j}\right\rangle_{X} \leqq 1 ;
\end{gathered}
$$

it can be seen from Eqs. [14] and [15] that the mean polydispersity and the effect of the interaction barrier are mutually dependent through particle size. ${ }^{2}$ When the flocculation proceeds according to the self-preserving theory, $\left\langle\sigma_{i j}\right\rangle_{X}$ will be relatively constant and identical to Swift and Friedlander's polydispersity factor ${ }^{3}$ derived analytically for a continuous size distribution.

When Eq. [13] is evaluated by a Smoluchowski-type plot $\left(1 / Y_{\infty}\right.$ versus $X$ as in Fig. 2), the predicted slope is $\left\langle\sigma_{i j} / W_{i j}\right\rangle_{X}$ and, consequently, various cases may be mentioned.

1. If there is no barrier to flocculation ( $W_{i j} \simeq 1$ ) and the particle size distribution is relatively monodispersed $\left(\sigma_{i j} \simeq 1\right)$, the slope will be unity and the reaction will proceed according to Smoluchowski's rapid floceulation theory.

2. If there is no barrier and the size distribution is polydispersed, the flocculation will proceed more rapidly with time since $\left\langle\sigma_{i j} / W_{i j}\right\rangle_{X}$ will be greater than unity owing to the Müller effect (1).

3 . If there exists an initial barrier of some

"Other possible expressions for the "means" of $\sigma_{i j}$ and $1 / W_{i j}$ at time $X$ are:

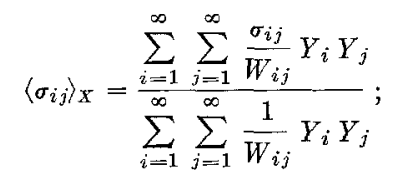

$$
\begin{aligned}
& \left\langle\frac{1}{W_{i j}}\right\rangle_{x}=\frac{\sum_{i=1}^{\infty} \sum_{j=1}^{\infty} \frac{1}{W_{i j}} X_{i} Y_{j}}{Y_{\infty}{ }^{2}} .
\end{aligned}
$$

${ }^{3}$ Refer to the polydispersity factor $A_{1}$ of Eq. 39 in reference 5 . magnitude, the flocculation will tend to slow down until the dispersion becomes kinetically stable as $\left\langle\sigma_{i j} / W_{i j}\right\rangle_{X}$ approaches zero.

4. Lastly, one has the intermediate situation in which the reaction is initially rapid and then slows down at a later time.

The expression describing the mutual interaction between floceulating particles is

$$
W_{i j}=2 \int_{0}^{\infty} \exp \left(V_{T(i, j)} / k T\right) \frac{d u}{(u+2)^{2}},
$$

where $V_{T(i, j)}$ is the total interaction potential between the $i$ th and $j$ th class particles shortest interparticle distance. If it is asand $u=2 H /\left(a_{i}+a_{j}\right)$, where $H$ is the sumed that the only rate process is the passage of the particles over the primary electrical barrier to flocculation (or coalescence), then

$$
\begin{array}{r}
V_{T(i, j)}=\frac{a_{i} a_{j}}{a_{i}+a_{j}}\left[e \psi_{0}^{2} \ln \left(1+e^{-\kappa^{H}}\right)\right. \\
\left.-\frac{A}{6 H}\left(\frac{\lambda}{\lambda+11.116 H}\right)\right],
\end{array}
$$

where $\epsilon$ is the dielectric constant of the medium, $\psi_{0}$ the surface potential, $\kappa$ the reciprocal double layer thickness, and $\lambda$ the wavelength of the atoms (7-10). A useful approximation of $W_{i j}(11)$ is

$$
W_{i j} \approx \frac{2 \pi^{1 / 2} \exp \left(V_{T(i, j) \max } / k T\right)}{\left(u_{\max }+2\right)^{2}\left(-V_{T(i, j) \max }^{\prime \prime} / 2 k T\right)^{1 / 2}}
$$

when the potential energy barrier from the $V_{T(i, j)}$ versus $u$ curve is sufficiently high. An examination of Eqs. [16] and [17] leads to the conclusion that smaller particles preferentially flocculate because of the presence of a smaller barrier than that for larger particles.

\section{METHOD OF CALCULATION}

The flocculation process for a dispersed system in water in which the primary repulsive barrier is electrical was simulated by the IBM 360 computer. Input data included $\mu=1 \mathrm{cp}, \epsilon=80, T=300^{\circ} \mathrm{K}, A=10^{-13}$ ergs, $\psi_{0}=6 \mathrm{mv}, \lambda=10^{-5} \mathrm{~cm}, \kappa=1.26 \times$ $10^{6}, 1 \times 10^{6}$, and $8 \times 10^{5} \mathrm{~cm}^{-1}$. Two initial size distributions were used: (a) monosized distribution where $Y_{i}=1$ and $v_{i}=1.1866$ $\times 10^{-12} \mathrm{~cm}^{3}$ (or $a_{1}=10^{-4} \mathrm{~cm}$ ) and (b) 
polydispersed sizes where $Y_{1}=0.819, Y_{5}=$ $0.173, Y_{10}=6.74 \times 10^{-3}$, and $Y_{15}=1.26$ $\times 10^{-3}$.

To solve Eq. [10] the stepwise integration procedure followed the predictor-corrector method of Hamming (12) in which the initial three-step values of $Y_{k}$ were obtained by the Runge-Kutta technique from the initial particle size distribution. The size of the largest aggregate was arbitrarily limited to $k_{\max }=100$ for the rapid and rapid-slow cases. Because this dimensional limitation and computational underflow tend to produce a loss of particles as the reaction proceeds, the total volume fraction was calcu- lated after each integration step; hence,

$$
\frac{\sum_{k=1}^{\infty} k Y_{k}(X)}{\sum_{k=1}^{\infty} k Y_{k}(0)}=1.0 \text {. }
$$

To compute $W_{i j}$, numerical integration by the Simpson method of Eq. [16] was used when $V_{T_{\max }}<5 k T$. When $V_{r_{\max }} \geqq 5 k T$, Eq. [18] was used. When the values of $W_{i j}$ were less than one, they were put into the computer program as unity.

\section{RESULTS AND DISCUSSION}

Rapid, Rapid-Slow, and Slow Flocculation. To define the terms-rapid, rapid-slow,

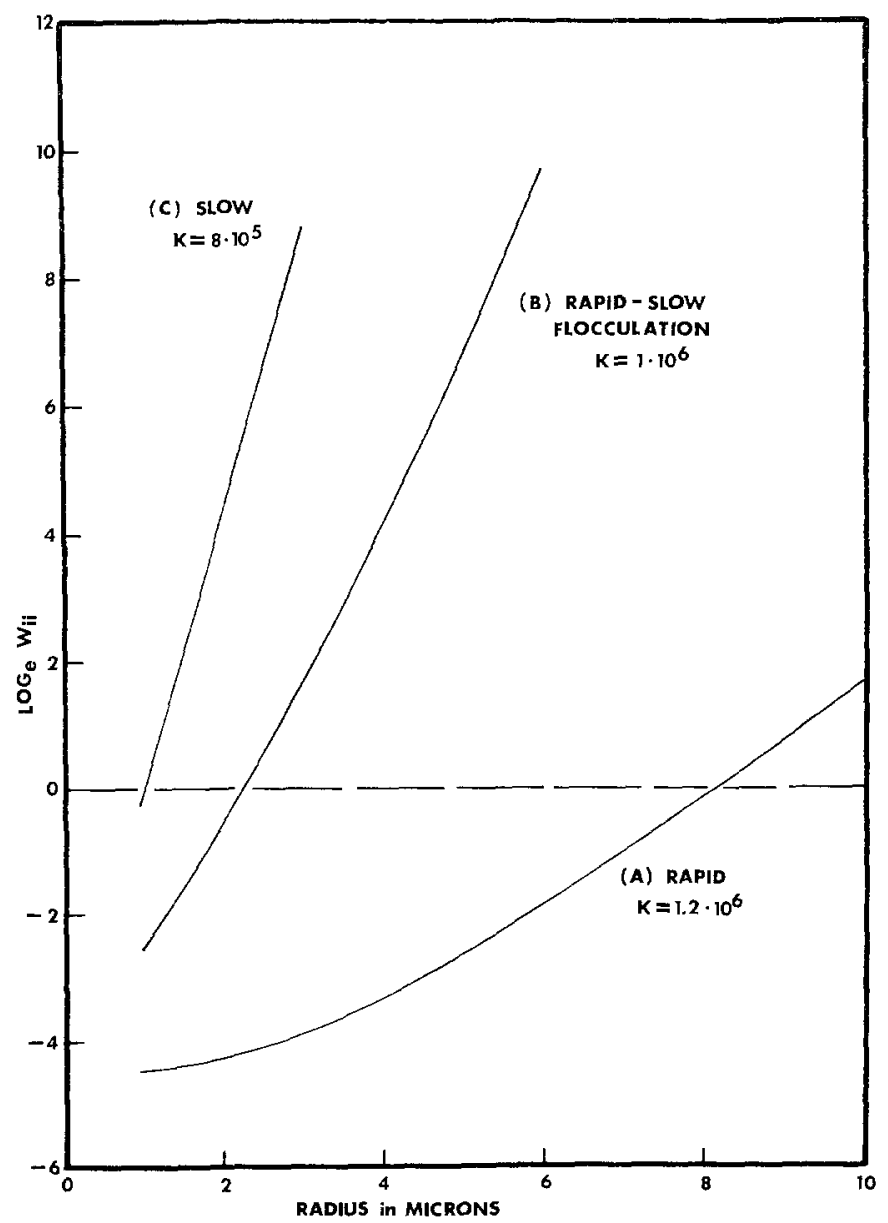

FIG. 1. Effect of size of two equal spherical particles on the stability factor $W_{i i}$ for various electrolyte concentrations $\kappa$. 
and slow flocculation, the stability factor as a function of the size of two equal interacting spherical particles for various electrolyte concentrations as shown in Fig. 1. The smallest particle size taken corresponded to the initial sizes that ranged from 1 to 2.47 microns.

When $k=1.2 \times 10^{6}($ Case $(\mathrm{A}))$, the $W$ values are less than one throughout the size region until $a=8 \mu\left(v_{i} / v_{1}=512\right)$. These values were taken as unity. In this case (A) the flocculation is rapid over most of the range of interest. Only at long times do the particle sizes become large enough and therefore the factor $1 / W$ small enough to appreciably reduce the collision rate. For case (B), in which $\kappa=10^{6}$, the intercept of the curve lies between $a=2 \mu\left(v_{i} / v_{1}=8\right)$ and $3 \mu\left(v_{i} / v_{1}=27\right)$. Here, according to the initial particle sizes chosen, the initial rapid rate is soon slowed down; thus, this reaction is defined as rapid-slow flocculation. In the last case (C) the $W$ values were always larger than unity in the region $a>1 \mu$; therefore, the reaction was slow for all times in this region.

The three sets of reaction parameters taken for this study provide typical cases to conveniently compare the particle size distribution changes with the stability factor. It is possible to obtain similar plots as shown in Fig. 1 for other surface potential and Hamaker constant values when $\kappa$ values are adequate, although the slope and intercept on the abscissa will change.

Flocculation of an Initially Uniform Size Distribution. The changes in the differential particle size distribution for an initially monodispersed dispersion are shown in Fig. 2 for three cases, i.e., rapid (A), rapid-slow (B), and slow (C) flocculation brought about only by changing the ionic strength of the system. Although the distributions appear to be graphically smooth, the distributions are discrete in nature. In the rapid case $(A)$, the flocculation proceeds rapidly, the heterogeneity of the dispersion increases, and the size spectrum broadens with positive skewness. Even in the absence of sedimentation, creaming, and/or adsorption of particles to the vessel wall, it is not expected that the size spectrum will broaden indefinitely in this case (A). As the particles grow in size

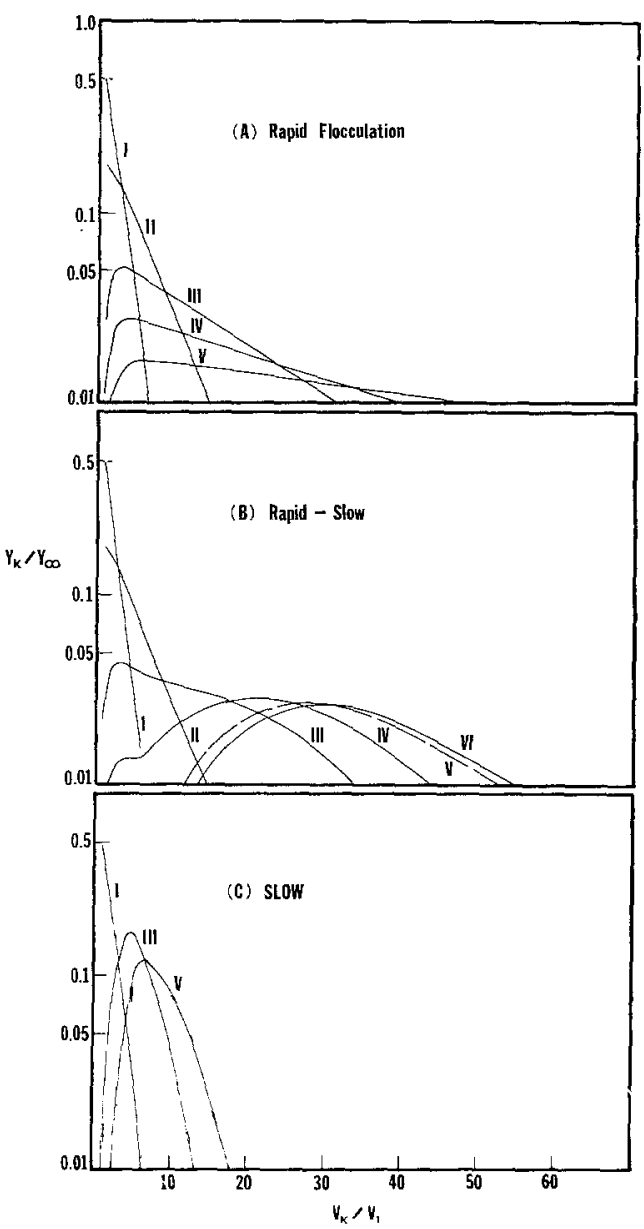

FIg. 2. Dimensionless particle size distribution as a function of time for an initially monodispersed size distribution for rapid flocculation $(A)$, rapid-slow $(B)$, and slow $(C)$ cases. Dimensionless time $X$ equals 1 (I), 8 (II), 16 (III), 32 (IV), 64 (V), and $80(\mathrm{VI})$. At least $98 \%$ of the mass was accounted for with the exception of case $(A)$ for $X=32(16 \%$ lost $)$ and $X=64(48 \%$ lost $)$.

by flocculation (or coalescence) the electrical barrier becomes increasingly important with particle size (see Eq. [17]). Consequently, although this is not seen in Case $A$, the flocculation rate will eventually become progressively slower. In the rapid-slow case (B), where $\psi_{0}=6 \mathrm{mv}, \kappa=1 \times 10^{6} \mathrm{~cm}^{-1}$, and $16 \leqq X \leqq 80$, this slowing down is more evident. A smooth size distribution slowly develops after the initial rapid flocculation period. Eventually, the distribution shifts to a larger mean particle size and be- 


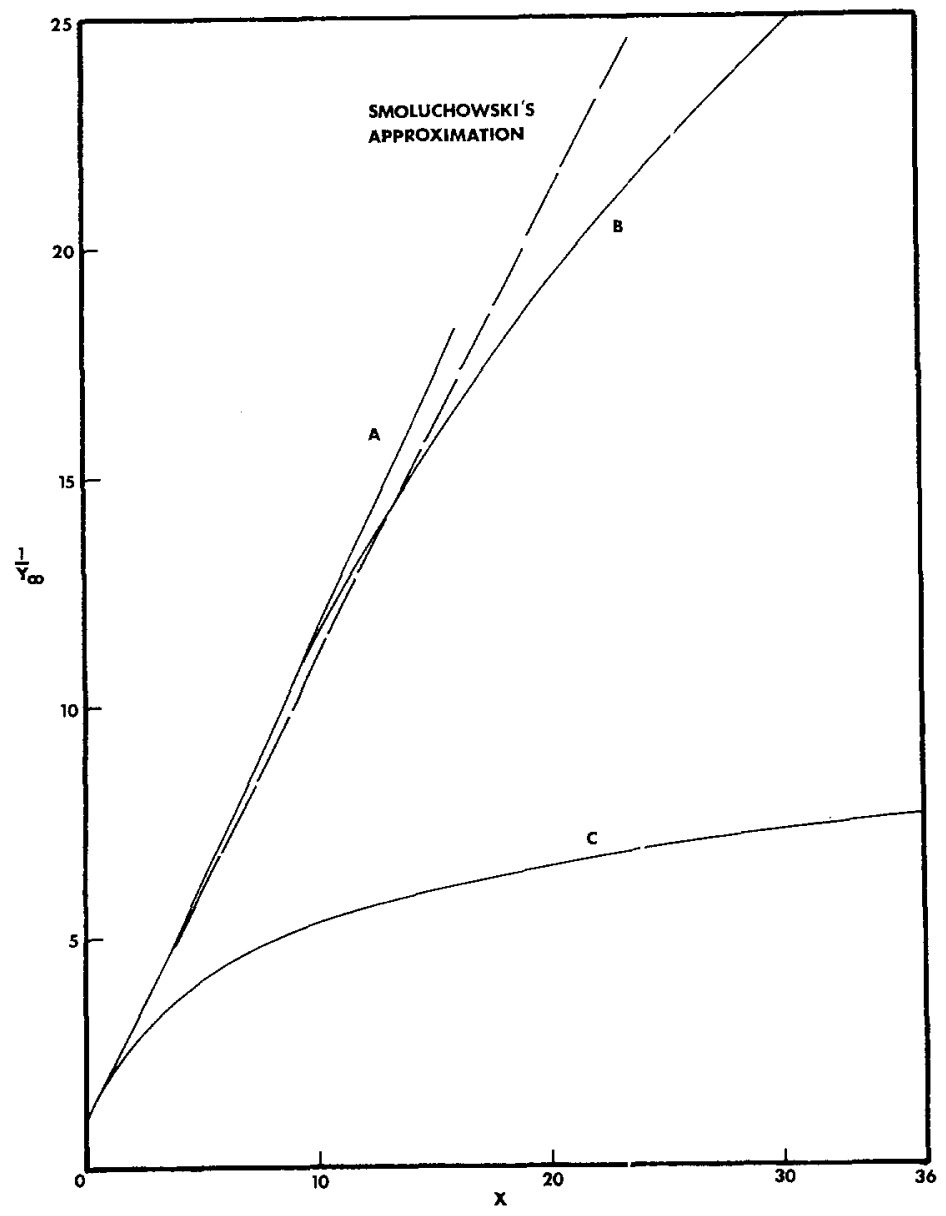

Fıg. 3. Smoluchowski-type plot from solution of dimensionless floceulation equation for an initially monodispersed size distribution for cases of rapid $(A)$, rapid-slow $(B)$, and slow $(C)$ flocculation.

comes narrower. Finally, in the slow case (C), there is relatively little skewing and broadening of the distribution at any stage and the distribution slowly shifts to larger sizes.

Figure 3 shows the Smoluchowski-type plot for the rate of change of the total number of particles according to the dimensionless form of the rate equation [13]. The three previously mentioned cases are compared with each other and the classical Smoluchowski theory for rapid flocculation. The curve for the rapid-slow case is the most general one and also the most interesting for discussion since it best describes the situation for a freshly prepared, finely dispersed suspension or emulsion. It is observed that the rate of flocculation (or coalescence when the surfactant coverage is only $5 \%$ to $10 \%$ ) is rapid and can even exceed the theoretical rapid Smoluchowski rate owing to the polydispersity effect of Müller. However, the rate progressively slows down until the dispersed system becomes kinetically stable.

The mechanism of flocculation is clearly shown in Figs. 4-6. The discussion in this section is confined to the initially monodispersed system. According to Smoluchowski's theory for rapid flocculation of a relatively monodispersed system, $\left\langle\sigma_{i j} / W_{i j}\right\rangle_{X}=$ $\left\langle\sigma_{i j}\right\rangle_{X}=\left\langle 1 / W_{i j}\right\rangle_{X}=1$. The $\left\langle\sigma_{i j} / W_{i j}\right\rangle_{X}$ versus $X$ plot describes the time dependency of the mean interaction of polydispersity and electrical barrier on the flocculation rate. It is observed in Figs. 4 and 5 that in the initial stages of flocculation polydispersity is 


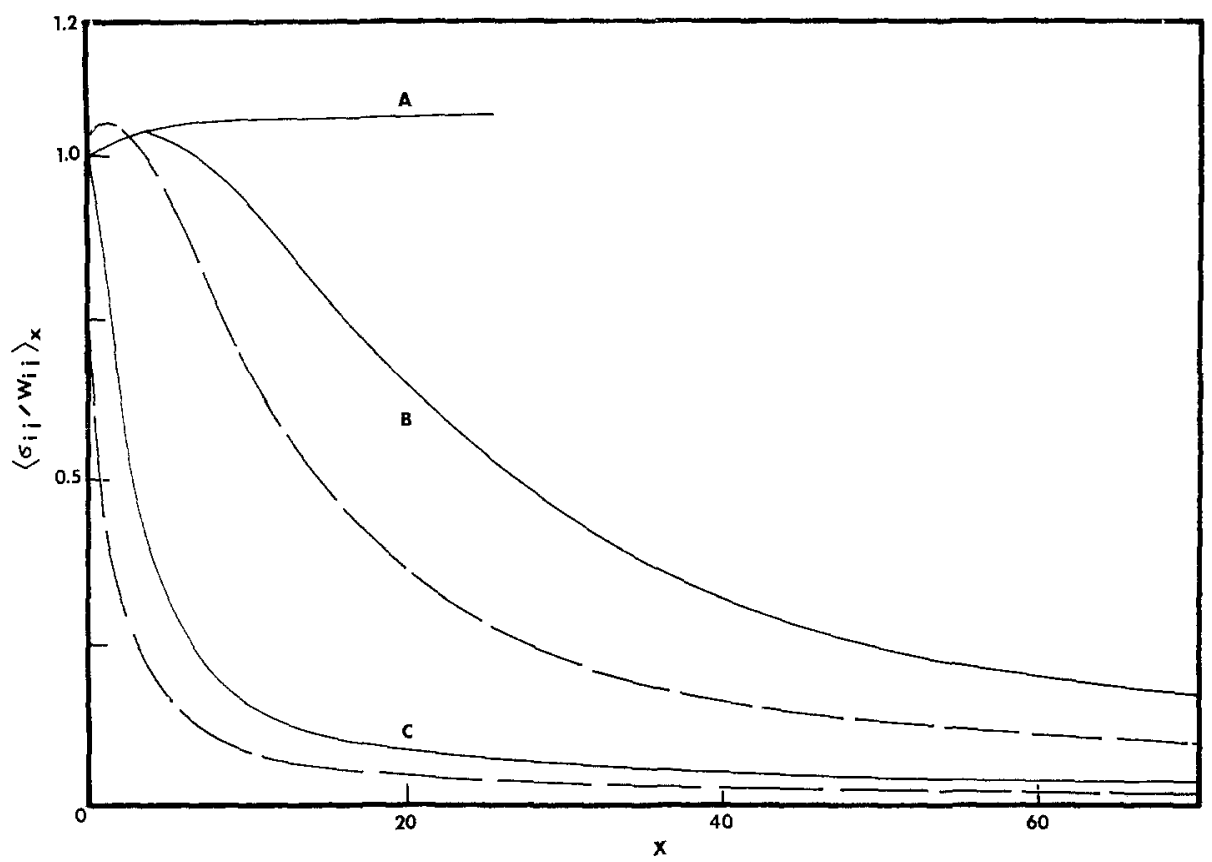

FIG. 4. Time dependency of the mean second-order rate constant from the numerical solution of Eq. [12]. Legend: initially monodispersed distribution--, initially heterodispersed distribution---.

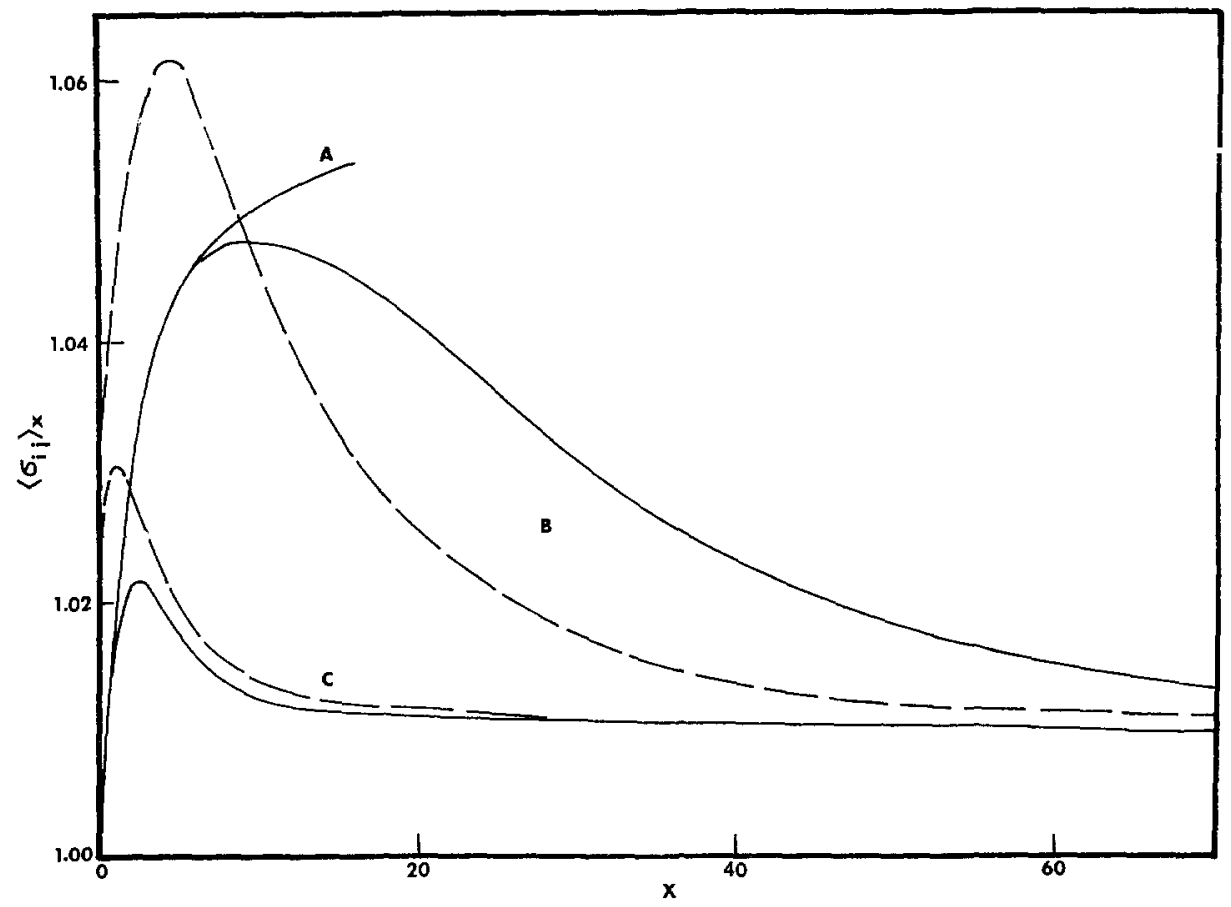

FIG. 5. Time dependency of the mean polydispersity. Legend same as Fig. 3 


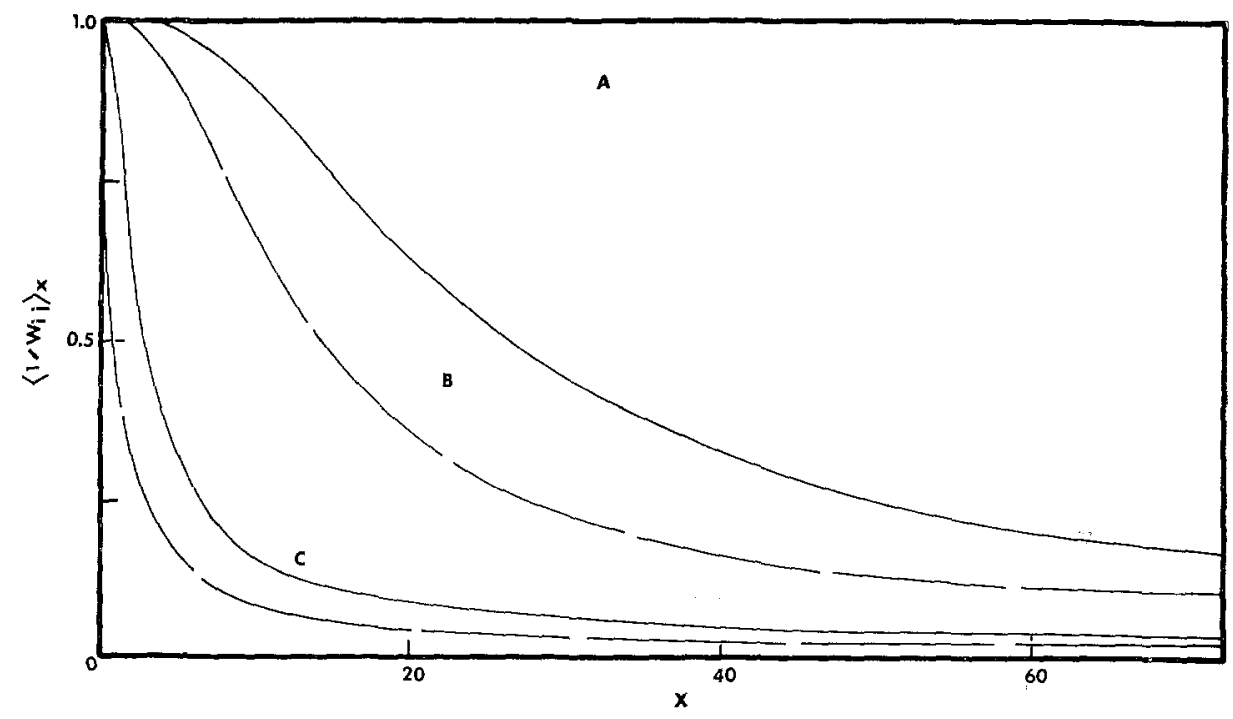

FıG. 6. Time dependency of the mean electrostatic energy interaction barrier

the predominant factor and this tends to increase the rate. Unless the mean particle interaction barrier is initially high (Case C), it does not exert its influence appreciably on the rate until some time later. The mean interaction barrier involves the energy barriers for all pairs of particles and, at small times, is weighted in favor of the small particles, for which the barrier is less than those between larger particles. From a mathematical viewpoint, the foregoing discussion can be explained by the fact that $\left\langle\sigma_{i j} / W_{i j}\right\rangle_{X}$ is the product of $\left\langle\sigma_{i j}\right\rangle_{x}$, a polynomial function of particle size, and $\left\langle 1 / W_{i j}\right\rangle_{X}$, an exponential function of particle size and energy barrier. In the later stages, the mean barrier is weighted towards the larger sizes and will have a twofold effect. Firstly, it will control the particle size distribution by preferential flocculation of smaller particles and, thereby, narrow the polydispersity. Secondly, it will decrease the over-all rate of flocculation. If we compare the rapid, rapid-slow, and slow flocculation cases (Figs. 3-6), the differences in the rates lie in the corresponding magnitudes of the mean polydispersity and the interaction barrier at the time.

Flocculation of an Initially Heterogeneous Size Distribution. In Figs. 4-8 the flocculation of an initially heterogeneous size distribution is shown in an analogous manner as that for the monodispersed situation. The initial distribution is positively skewed and rather narrow, that is, about $82 \%$ of the total number of particles are 1.0 micron radius, the rest are equal or greater than 1.71 micron, and the size range is 1 to 2.47 micron radius. Since Hidy (2) had already treated this initial distribution for rapid flocculation, only the rapid-slow and slow flocculation cases are shown. It is observed in Fig. 7 that the multiple peak distribution of the rapid-slow case at somewhat early times eventually smooths out to a distribution that maintains its general shape with time. For the slow case the similarity of the spectrum develops even more slowly.

Although the effect of polydispersity and interaction barrier on the rate is qualitatively the same for an initially monodispersed and heterodispersed size system, there is a quantitative difference between them relative to time for the same flocculating conditions. In the rapid-slow case the polydispersity $\left(\left\langle\sigma_{i j}\right\rangle_{X=0}=1.023\right)$ and the interaction barrier $\left(\left\langle 1 / W_{i j}\right\rangle_{X=0} \lesssim 1\right)$ cause the initial rate to be $2 \%$ faster than that for the initially monodispersed distribution. (Fig. 4). After $X=3$, the rate becomes slower and approaches the same rate as the latter. In the slow case the strong influence of the mean interaction barrier of the heterodispersed distribution significantly delays the 


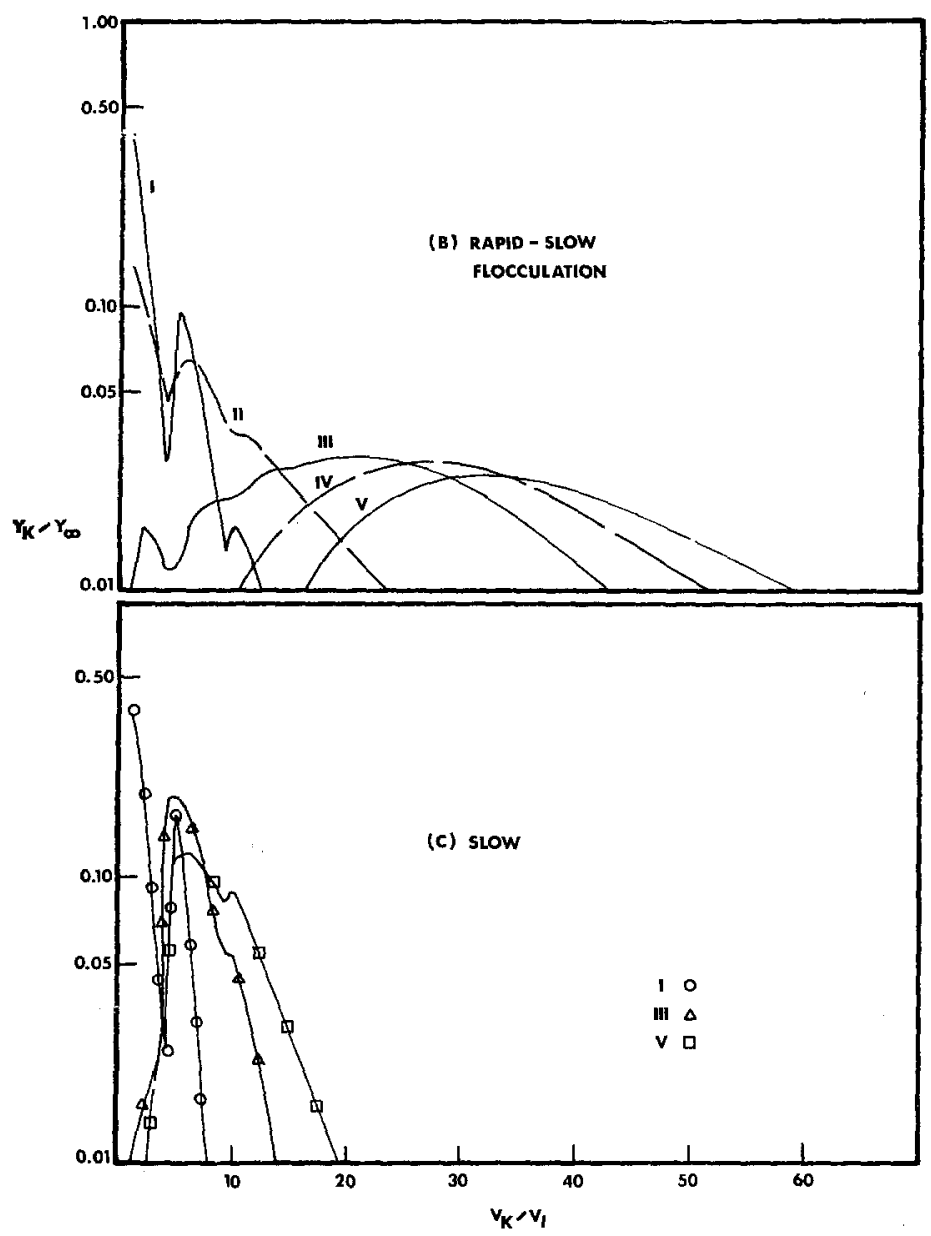

Fig. 7. Dimensionless particle size distribution as a function of time for an initially heterodispersed size distribution. Legend same as Fig. 2. All mass was accounted for.

course of flocculation. As a result the development of similarity between the two initial distributions takes a great deal longer.

Analysis of the Self-Preserving (Similarity) Spectrum. The self-preserving dimensionless size distribution function $\psi(\eta)$ for a discrete distribution is defined by Hidy as

$$
\psi\left(\eta_{i}\right)=\frac{n_{i} \phi}{n_{\infty}^{2} V_{1}}=\frac{Y_{i} \phi}{Y_{\infty}^{2} N_{0} v_{1}}
$$

where the volume fraction is

$$
\phi=\Sigma i n_{i}=N_{0} v_{1} \Sigma i Y_{i}
$$

and the dimensionless volume $\eta_{i}$ is $\Sigma$

$$
\eta_{i}=\frac{i v_{1} n_{\infty}}{\phi}=\frac{i v_{1} N_{0} Y_{\infty}}{\phi}
$$

According to the theory, a plot of $\psi\left(\eta_{i}\right)$ versus $\eta_{i}$ should show an asymptotic behavior of the particle distribution becoming eventually independent of time and the initial distribution.

For the case of the initially monodispersed distribution in Fig. 9, the distribution of the similarity function approashes an asymptotic behavior after some period of floceulation. Whether the flocculation is rapid, rapidslow, or slow, the spectrum reaches a selfpreserving form more quickly in the upper range of $\eta$ than in the lower ranges. The data in Fig. 9(A) for rapid flocculation compare well with the numerical results by Hidy, ${ }^{4}$

$$
\begin{aligned}
& { }^{4} \text { According to Hidy's notation }(2), \tau=3 ; \\
& X=4 \tau_{\text {Hidy }} \text {. }
\end{aligned}
$$




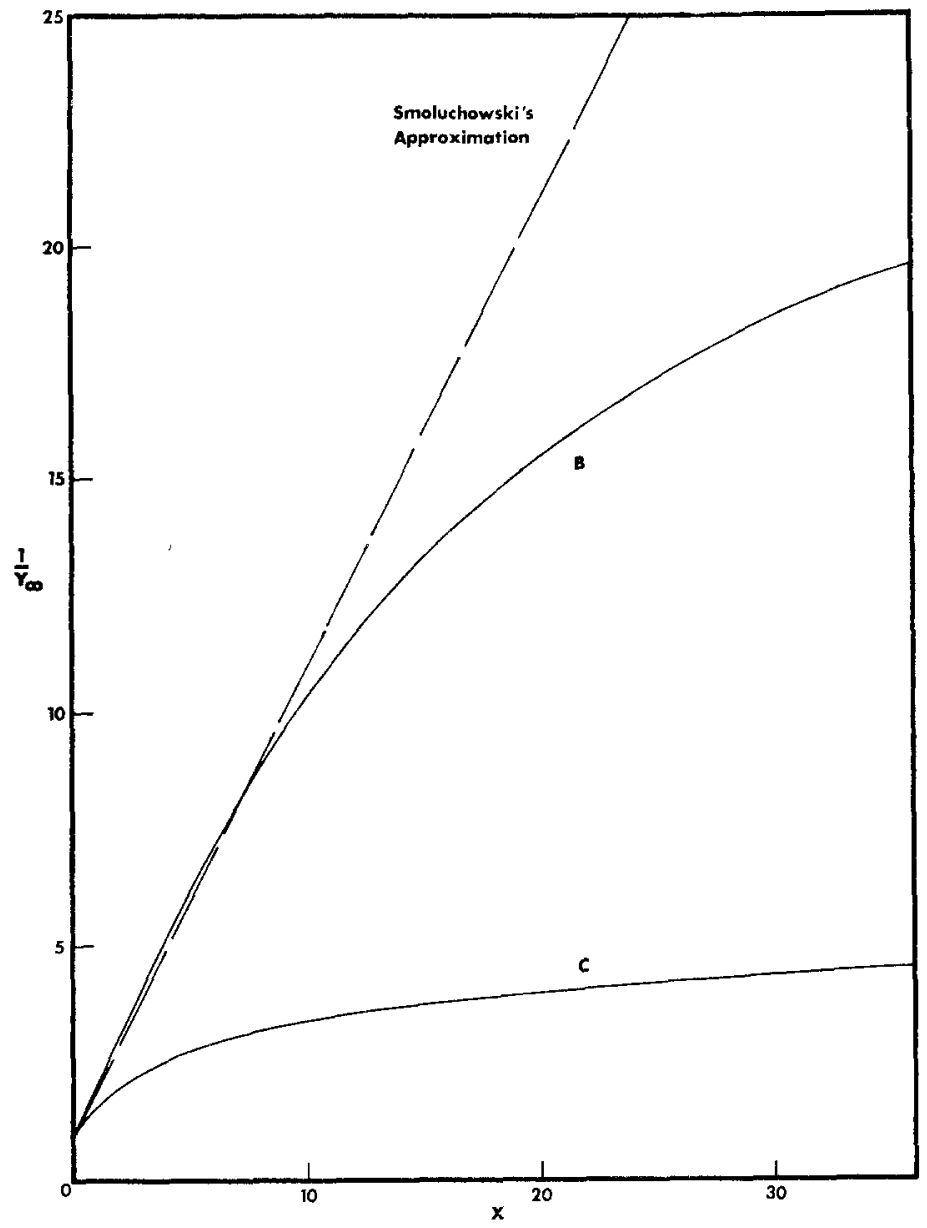

FIG. 8. Smoluchowski-type plot for an initially heterodispersed size distribution

i.e., $\psi(\eta)$ develops after $X=12$ and its maximum lies at $\eta \approx 0.1$. Any slight discrepancies may be due to the differences in computer schemes used in this study. On the other hand, the results of the slow case (C) are different from those of the rapid one (A). The former shows that the shape of the spectrum is markedly changed, $\psi(\eta)$ develops after $X=8$, and its maximum is at $\eta \approx 0.8$. A comparison between the rapid-slow case (B) and the other two shows that the distribution for the rapid-slow case first approaches the asymptotic spectrum of the rapid case, then, at later times, undergoes a marked change, and finally asymptotically develops the distribution of the slow case. Accordingly, if the rapid flocculation (A) is carried out long enough so that the interaction barrier becomes appreciable (Fig. $1 A$ ), the spectrum for the slow flocculation condition may be attained. Swift and Friedlander used a mixture of two unimodal emulsions in their flocculation experiments. However, their results show the same trend of a marked shift and change of $\psi(\eta)$ after a period of time to larger values of $\eta$ as that of the rapid-slow flocculation case of an initially unimodal distribution.

In accordance with the similarity theory, the initially polydispersed system approaches the same distribution as the monodispersed system (Fig. 10), provided that the flocculation is carried out long enough. In case $(B)$ three distinct peaks at $\eta=0.1$, 0.4 , and 0.8 when $X=8$ are found at early times. These peaks eventually disappear 


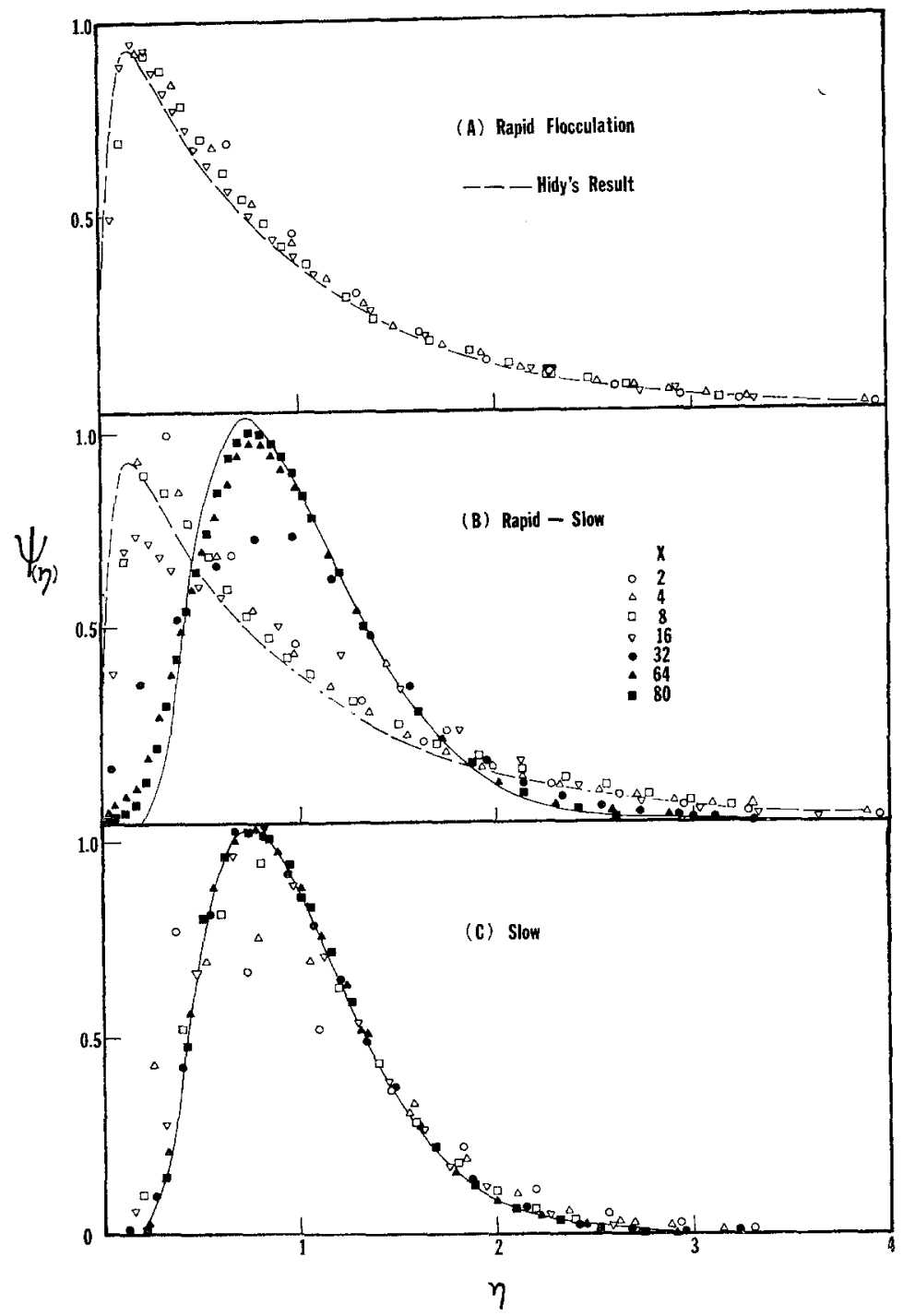

FIG. 9. Self-preserving size distribution $\psi(\eta)$ for an initially unimodal distribution for rapid flocculation $(A)$, rapid-slow $(B)$, and slow $(C)$ cases. The broken curve is Hidy's result for rapid flocculation and the solid curve is the asymptotic distribution for slow flocculation.

with the development of a single $\psi(\eta)$ maximum at $\eta=0.8$ and a smooth asymptotic distribution. (At relatively early periods of the flocculation, i.e., $X=16$, the distribution was self preserving for $\eta>1.3$.) Comparing case (C) with (B) in Fig. 10, one observes a qualitative difference in the development of the similarity distribution. It is expected that both the distributions will be the same at sufficiently long times.

The time required to assume the selfpreserving form was greatest for the poly- dispersed system. In contrast to the monodispersed system, the time required for the polydispersed system was much greater for the slow flocculation than the rapid-slow case It is interesting that the $\psi(\eta)$ for all three flocculation cases for an initially monodispersed system became time independent when their particle size distribution changes with time were smooth and similar in shape (Fig. 1), and simultaneously their mean rate constant, polydispersity, and interaction barrier (Figs. 2-4) became essentially constant 


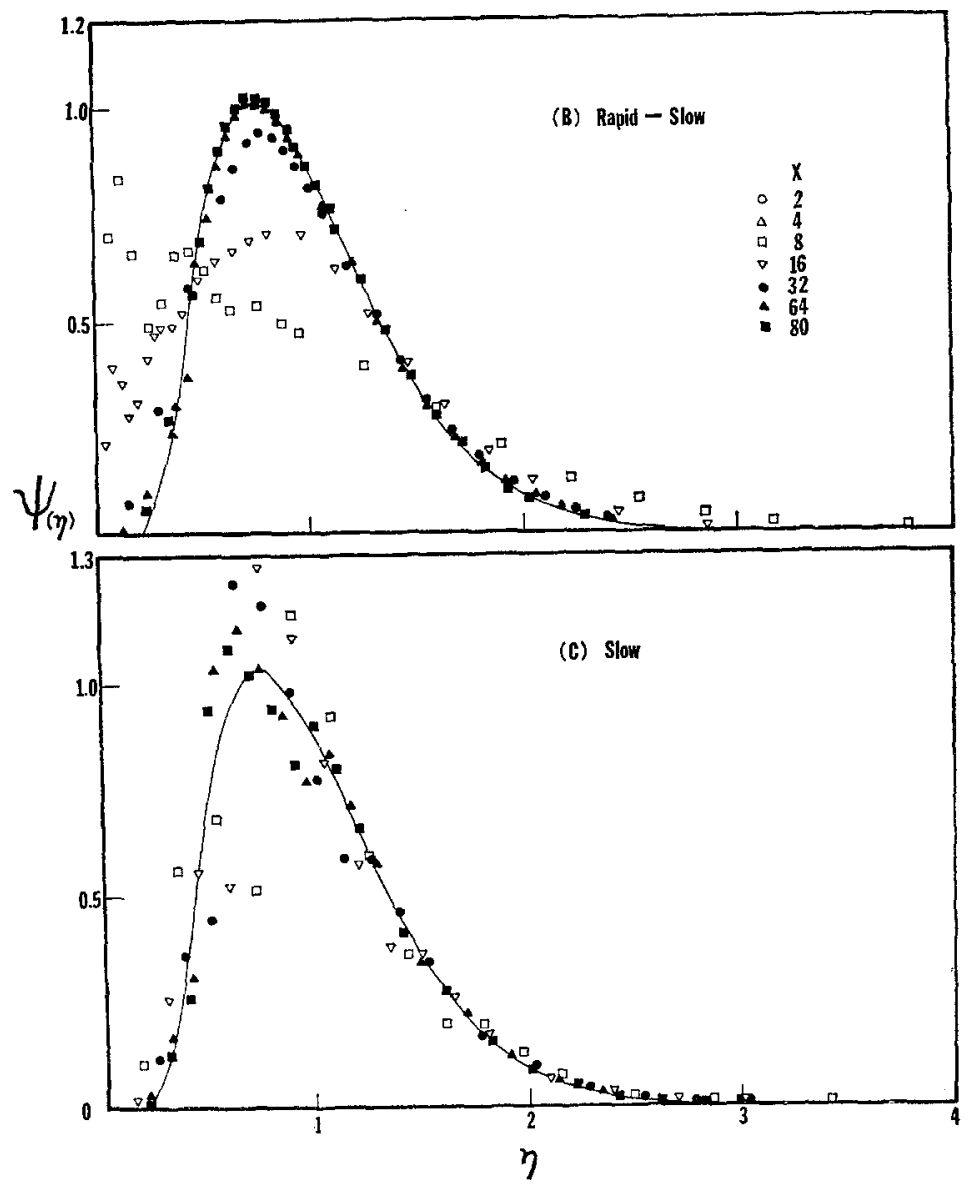

FIg. 10. Self-preserving size distribution $\psi(\eta)$ for an initially polydispersed distribution. The solid curve is the self-preserving distribution for an initially unimodal distribution taken from Fig. 9.

with time. However, these observations did not coincide with the flocculation cases for an initially polydispersed system. Although the mean rate constant and other quantities (Figs. 2-4) were somewhat time independent, $\psi(\eta)$ was not constant until the particle size distributions (Fig. 7) were smooth and similar in shape.

The effect of the degree of initial polydispersity and interparticle energy barrier on the time required and the form of the similarity function seem worthy of investigation. Since $W_{i j}$ is not a homogeneous function of particle size, it is not expected that the self-preserving spectra exist according to the exact theory of Friedlander and Wang (6). However, in certain cases quasi-self-preserving spectra seem to exist even though $W_{i j}$ is not homogeneous (13). The example for slow flocculation in this study appears to be another case. As shown by Wang and Friedlander (13) in the slip correction probIem, there can exist different self-preserving functions.

\section{CONCLUSION}

In conclusion the theoretical predictions of the changes in the particle size distribution in emulsions and suspensions may be directly compared with experimental data obtained from an electronic resistance multichannel particle size analyzer (14). The general method of numerical analysis should be easily adaptable to cases involving other forces of repulsion between particles such as steric (entropic) repulsion. It is believed that these techniques should provide a 
powerful handle on various complex problems in dispersed systems.

\section{REFERENCES}

1. KRuYt, H. R., "Colloid Science," Vol. I, Chapter 7. Elsevier, Amsterdam, 1952.

2. Hidy, G. M., J. Colloid Sci. 20, 123 (1965).

3. Hidy, G. M., and LiLlu, D. K., J. Colloid Sci. 20, 341 (1965).

4. Rice, C. L., AND Whitehead, R., J. Colloid Sci. 23, 174 (1967).

5. Swift, D. L., and Friedlander, S. K., $J$, Colloid Sci. 19, 621 (1964).

6. Friedlander, S. K., and Wang, C. S, $J$. Colloid Sci. 22, 126 (1966).

7. Ho, N. F. H., AND Higuch, W. I., J. Pharm. Sci. 57, 436 (1968).
8. Verwey, E. J. W., AND OVERBEeK, J. T. G., "Theory of the Stability of Lyophobic Colloids," p. 139. Elsevier, Amsterdam, 1948.

9. HogG, R., Healy, T. W., and Fuerstenau, D. W., Trans. Faraday Soc. 62, 1638 (1966).

10. Schenkel, J. H., and Krtchener, J. A., Trans. Faraday Soc. 56, 161 (1960).

11. Reerink, H., and Overbeek, J. T. G., Discussions Faraday Soc. 18, 74 (1954).

12. RaLSTON, A., AND WILF, H., "Mathematical Methods for Digital Computers." Wiley, New York, 1960.

13. Wang, C., ANd Friedlander, S. K., J. Colloid and Interface Sci. 24, 170 (1967).

14. Ho, N. F. H., ANd Hxguchi, W. I., J. Pharm. Sci. 56, 248 (1967). 